\title{
Demonstration of Calcium Ion Distribution in Calcifying Cells -X-ray Microanalysis and Electron Spectroscopic Imaging after Fixation with NHA-Containing Fixative and Microwave Irradiation-
}

\author{
Vinci Mizuhira ${ }^{1 *}$, Hiroshi Hasegawa ${ }^{2}$, Takumi Akagi ${ }^{3}$ and Noriyuki Nagai $^{3}$ \\ 'Department of Cell Biology and Anatomy, Tokyo Medical and Dental University, Bunkyo-ku, Yushima, Tokyo \\ 113-8510, ${ }^{2}$ Research Institute for New Drags, Shionogi Co. Ltd., Futaba-cho, Toyonaka, Osaka \\ 561-0825 and ${ }^{3}$ Department of Oral Pathology, School of Dentistry, University of Okayama, \\ Shikada-cho, Okayama, 702-8031
}

Received for publication April 9, 1998

\begin{abstract}
We have seen reporting on the calcium ion distribution in cells and tissues with $X$-ray microanalysis (EDX) since 1978 [9] and with electron energy-loss spectroscopy (ESS) imaging since $1983[10,17]$, under various fixing conditions [12-16, 18-24]. Almost ten years ago, we tried a fixative containing NHA ( $N, N$-naphthaloylhydroxamine) as a chemical precipitant for $\mathrm{Ca}^{2+}$ ions under microwaved conditions [12, 19]. However, the data did not seem to be as sensitive or useful, under conventional electron microscopy and computerized $X$-ray microanalysis, compared to the two-step replacement method combined with microwave fixation using potassium (K) oxalate followed by potassium (K) antimonate, for the detection of $\mathrm{Ca}^{2+}$ ions [12, 19-21]. Recently, however, we used ESS to analyze same tissue sections which were fixed with NHAcontaining fixative with microwave fixation ten years previously. We discovered with

ESS analysis that NHA is a sensitive and ideal chemical reagent for calcium ion detection in a cell giving the same view as that of frozen dried or freeze-substituted tissue sections. Calcium was clearly seen and widely distributed in developing chondroblast endoplasmic ultrastructures: ribosomes, cell and endoplasmic membranes, mitochondria, Golgi, and nucleus. However, free mesenchymal cells which were not involved in calcifying functions had only a very small amount of calcium. Calcium was widely distributed in the lacunae of the surrounding space of the chondroblasts and inmature matrix, but the exoplasm of the chondroblasts was almost calcium-free. Other elemental images in the young chondrocytes or osteoblasts, i.e., P.L, S.L, O.K, and N.K, were seen clearly superimposed on the cell ultrastructures, i.e., the cell membranes, ribosomes, endoplasmic reticula, mitochondria, Golgi, and nucleus [24].
\end{abstract}

Key words: Ca distribution in Calcifying cells, Microwaved with NHA fixative, ESS-imaging

\section{Introduction}

Calcium $\left(\mathrm{Ca}^{2+}\right)$ ions in living cells and tissues are distributed, especially in developing calcifying tissues, where there are a large number of $\mathrm{Ca}^{2+}$ ions; on the other hand, only a very small amount of $\mathrm{Ca}^{2+}$ ions is distributed in the membraneous structures of the nerve synaptic terminals or

Correspondence to: Vinci Mizuhira, Prof. Emeritus, Tokyo Medical and Dental University, Dept. Cell Biology and Anatomy, Bunkyo-ku, Yushima, Tokyo 113-8510, Japan.

* Present Address: 3-20-13, Kichijoji Honcho, Musashino, Tokyo 180-0004, Japan. sarcoplasmic cisternae in the muscular cells, where they play important roles in very important physiological functions, such as the transmission of nerve stimuli in the nerve terminals, and the contraction of muscular cells. But morphological study of calcium ions is not easy.

They can be studied by various methods under the electron microscope. (1) Fresh tissue can be rapidly frozen, cut into frozen ultrathin sections, and then analyzed using a computerized electron beam X-ray microanalysis with a dispersive type X-ray detector (EDX) $[9,10,16,17,19,21]$ or electron energy-loss spectroscopy (ESS) imaging [10]; (2) fresh tissue blocks can be freezesubstituted with cooled acetone or ether, microwaved for 
2-3 $\min$ in an Acroleine vapor atmosphere $[9,10,19]$, and analyzed under EDX or ESS after embedding in Epon or a low viscosity resin, such as Spurr; (3) inorganic chemical reagents can produce fine, inorganic, insoluble, and electron-opaque precipitates with $\mathrm{Ca}^{2+}$ ions in the cell, and there can be analyzed with EDX or ESS-imaging [12-16, 18-24]; (4) as a control, the chemical nature of the precipitates can be confirmed by analysis with EDX and ESS, or by calcium substitution in the section with EDTA or EGTA solution $[10,12,16,22]$. The reliability of method (4), especially with EDTA or EGTA method seems very low. This is because, they extract not only calcium, but also other elements, such as chemical precipitants, antimony, and others. Methods (1) and (2) seem good theoretically $[9,10,21]$, but they have, especially method (1), some technical drawbacks. Method (2) seems rather good with a few technical drawbacks $[9,10,17]$. For this reason, we have used (3)-method often [12-16, 18-24] combined with microwave irradiation (MWI) [12-16, 1824]. Microwave fixation produces almost perfect biological fixation effects within a very short time, and the data are identical to those of the rapid frozen dried or freezesubstituted fixation methods [12-16, 18-24].

We developed a new method for fixation and staining of biological specimens, which gives excellent results using a computerized household microwave oven $[12,16,19$, 20]. We investigated the mechanism of fixation with MWI, i.e., why MWI enabled aldehyde to penetrate into the tissue block so quickly and homogeneously within a few seconds, with thaw mount cryomicroautoradiography using ${ }^{3} \mathrm{H}$-formalin as a tracer. With MWI treatment for only $20 \mathrm{sec}$, prominent developed silver grains were distributed homogeneously in thaw mount cryosections, but not in conventional cryosections immersion-fixed for $20 \mathrm{sec}[12,13,16,19,20]$. We also confirmed why MWI enabled the staining materials, uranyl acetate and lead hydroxide solutions, to penetrate into sections quickly and homogeneously by computerized EDX pulse intensity ratios of $\mathrm{U} \cdot \mathrm{L} \alpha$ and $\mathrm{Pb} \cdot \mathrm{M} \alpha \quad[12,16,19-21]$. We described the applications of MWI to conventional light and electron microscopy, including histochemical and cytochemical immunocytochemistry, electron microscope autoradiography, and the distribution of ionic elements, such as calcium, using computerized EDX and ESS imaging [12-16, 18-24].

Using tannic acid (TA) with aldehyde fixative, good, rapid fixation was obtained when MWI was used [11-16, 18-24]. Combined TA/MWI fixation and double electron staining/MWI methods have produced exellent, perfect and rapid fixation and staining of proteins and small, soluble peptides, and even enkephaline [11-16, 18-24]. In such conditions, it is easy to obtain images of nerve synaptic membranes, ribosomes and associated messenger RNA filaments, cytoplasmic skeletons, and the ATP-synthesize particles on the surface of the mitochondrial cristae, and connections between the microtubules or neurotubules and the outer surface of the mitochondria could often be observed [12, 14-16, 18-23].

For studying the distribution of calcium ions $\left(\mathrm{Ca}^{2+}\right)$ in living tissue and cells, various fixation methods have been tried since Komnick [4], Legato [7], Mata [8], Ohara [27], Simson [36] and others $[9,12,19,21]$. We devised a new technique to detect the localization of $\mathrm{Ca}^{2+}$ ions in tissue and cells which was modified from Bogers et al., [2, 3, 39]. To detect the $\mathrm{Ca}^{2+}$ ions in tissue and cells using a two-step fixation method, we used aldehyde fixative containing $30-60 \mathrm{mM}$ of K-oxalate (Boger's original method used $90 \mathrm{mM}$, this often damaged the ultrastructures, especially in the case of nervous tissue, so we used $30 \mathrm{mM}$ for nervous tissues, and $40-60 \mathrm{mM}$ for other tissues) with MWI for $35 \mathrm{sec}$ at room temperature, left the specimens in the same fixative for $60 \mathrm{~min}$ at room temperature, and then postfixed them with $1-2 \%$ osmium tetroxide containing $1-$ $1.5 \% \mathrm{~K}$-antinomate (Boger's original method used $2 \% \mathrm{~K}$ antimonate) $[12,14-16,18-21,23,37,38]$. Microwave fixation produced perfect and rapid fixation with homogeneous infiltration of the fixative solution [12-16, 19-22]. Therefore, fines and more hogeneous chemical precipitates can be observed in the cell, than with the conventional infiltration fixation methods. We also succeeded in calculating the atomic binding ratios (AT, \%) and atomic weight ratios (WT, \%) of the precipitates of calcium antimonate, and concluded that the precipitates are composed of calcium and antimony by analysis with computerized EDX after strict calibration for the X-ray detector for $1 \mathrm{hr}[12,14-16,18,20,21,37,38]$. We also demonstrated the distribution image of calcium and other elements by the ESS-imaging method [14-16, 18, 23]. However, there remained some problems, such as sensitivity, the mechanism of the formation of precipitates, the size of precipitates and their sensitivity, the electron opacity, and the difference between the frozen dried and freeze-substitution methods.

Our two-step method was very reliable for detecting calcium ions as a chemical fixation method followed by the frozen method, but we still wished to find a method as good as the frozen one. We confirmed, very recently, that fixation with a fixative containing NHA (N,N-naphthaloylhydroxamine) (Fig. 1-1, 2 and Fig. 3A) under microwaved conditions and analyses with ESS-imaging brought us almost perfect fixation of calcium ions in cells and tissues, giving the same results as the frozen or freezesubstituted method [24]. Originally, the sensitivity of this NHA toward $\mathrm{Ca}^{2+}$ ions was thought to be $0.1 \mu \mathrm{g} / \mathrm{ml}$ [1], and precipitates with calcium were so fine and electron transparent that they could not be recognized with conventional electron microscopy except outside the cell spaces. NHA also made insoluble precipitates with peptides and proteins [1]. This chemical nature is very useful to fix cells and tissues in combination with tannic acid aldehyde fixative $[11-13,19-22]$. Success was achieved by the application of ESS-imaging analysis after microwaving the fixation using fixative containing NHA. 


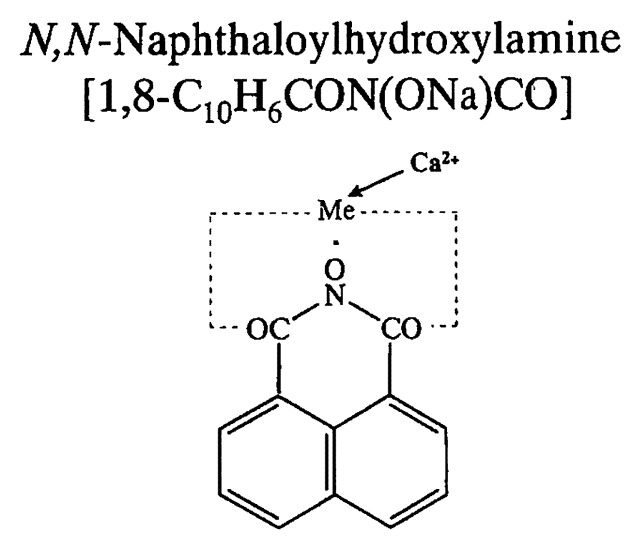

(Sensitivity for $\mathrm{Ca}^{2+}, 0.1 \mu \mathrm{g} / \mathrm{ml}$ ) (G.Beck, 1951)

Fig. 1-1. Chemical formula and structure of NHA and its detecting sensitivity. The heavy earth metals $\mathrm{Ca}, \mathrm{Bi}, \mathrm{Cd}, \mathrm{Pb}$, and others form insoluble precipitates, and peptides and proteins and some sugars also form precipitates with NHA [1]. For the calcium ions, the sensitivity of NHA is very high, $0.1 \mu \mathrm{g} / \mathrm{ml}$. The density of $\mathrm{Ca}^{2+}$ ions is very thin, and there appear very fine precipitates dispersed in the cell cytoplasm, but if the intensity is high, larger stick-shaped crystals are formed (see, Figs. 5, 6). The electron diffraction pattern obtained from a crystal in the section is shown in Fig. 6 (insert).

\section{Materials and Methods}

We used developing bone, mandibular bone, parietal bone, or rib cartilage anlages obtained from newborn rats (Sprague-Dawley), just after birth (up to two days after birth) $[12,19]$.

Small tissue blocks were cut from anesthetized baby rats, cut into small pieces in a drop of NHA-containing fixative on a plastic plate, and microwaved (Nissin EM MWF-2500/100W was used*) for $35 \mathrm{sec}$ at room temperature in a water bath $[12,19,24]$. After microwave irradiation (MWI), the tissue blocks were left in the same fixative for $60 \mathrm{~min}$ at room temperature, as described before [12-16, 18-24].

The fixative solution was composed of $2 \%$ paraformaldehyde, $0.5 \%$ glutaraldehyde, $0.1 \%$ tannic acid (TA), and $0.1-0.5 \mathrm{mM} \mathrm{NHA}^{* *}$ ) in $0.1 \mathrm{M}$ sodium cacodylate buffer at pH 7.2. Postfixation was done with $1 \%$ osmium tetroxide in cacodylate buffer at $\mathrm{pH} 7.2$ for $60 \mathrm{~min}$ at room temperature. In some cases, the postfixation was skipped for ESS-imaging analysis. The tissue blocks were embedded in Epon after dehydration to obtain consistent data.

For conventional electron microscopy, 50-60 nm sections were electron stained with MWI [12, 14-16, 19-22]

\footnotetext{
* Nisshin EM Co. Ltd., Fax: (03)-3353-2888; Araki-cho, Shinjuku, Tokyo, 160-0007. ${ }^{* *}$ The Chemical formula of NHA (N,NNaphthaloylhydroxylamine) is: $1,8-\mathrm{C}_{10} \mathrm{H}_{6} \mathrm{CON}(\mathrm{ONa}) \mathrm{CO}$, and its molecular weight is 235.2. We used Sigma products (Fig. 1-1, 2).
}

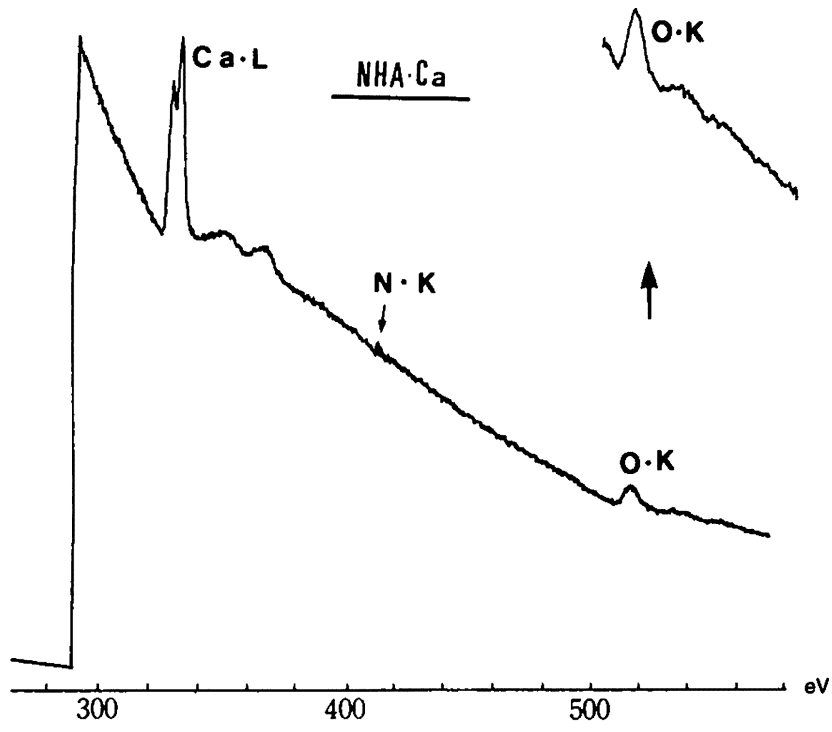

Fig. 1-2. ESS-spectra obtained from the Ca-NHA crystals in a section. The $\mathrm{Ca} \cdot \mathrm{L}_{2,3}(355 \mathrm{eV}, \mathrm{Max}) \mathrm{O} \cdot \mathrm{K}(540 \mathrm{eV})$, and $\mathrm{N} \cdot \mathrm{K}(410 \mathrm{eV})$ ESS-spectral peaks can be clearly seen. The carbon C.K $(284 \mathrm{eV}$, $\left.\mathrm{Max}^{*}\right)$ is not shown here. See Fig. 2-1. ${ }^{*}$ (Max: maximum value of electron energy-loss spectrum)

and coated by carbon evaporation. For the EDX analysis, unstained 50-60 nm sections were picked up on a nylon grid, and examined with a computerized EDAX PV-9800 (Japan Philips Co. Ltd.) fitted to a JEM-1200EX transmission electron microscope using scanning (SEM) and scanning transmission (STEM) mode after careful calibration of the dispersive type X-ray detector, as described previously $[12,14-16,18-23,37,38]$.

For ESS-imaging, sections were cut at $30 \mathrm{~nm}$ thickness with a diamond knife [10, 18, 23], and picked up on a 400-600 copper grid mesh. The $30 \mathrm{~nm}$ ultrathin sections were obtained as shown in Fig. 2-2. It is difficult to recognize the superultrathin sections at this thickness, because the sections floating on the water of the knife boat are so thin, that they do not show any interference color. However, if two or three silver-colored $50 \mathrm{~nm}$ sections are cut first, then some $30 \mathrm{~nm}$ sections, and then more $50 \mathrm{~nm}$ sections, the $30 \mathrm{~nm}$ sections can be rcognized as transparent spaces between the silver colored $50 \mathrm{~nm}$ sections (Fig. 2-2). When making freeze-substitution ultrathin sections, the ethylenglycole or glycerin should be used instead of water in the knife boat because the ionic elements will be leached out in the water [10].

Unstained dried sections were observed under a Carl Zeiss LEO-912 Omega-type ESS electron microscope (Okayama University) at $100 \mathrm{kV}$ accelerating voltage.

In ESS-imaging analysis, it is neccesary to use thinner sections, so we used sections of about $30 \mathrm{~nm}$ in thickness. Thinner sections provide more elemental information as shown in Fig. 2-1.

Before the elemental analysis of the biological sections, continuous energy-loss spectra obtained from the 


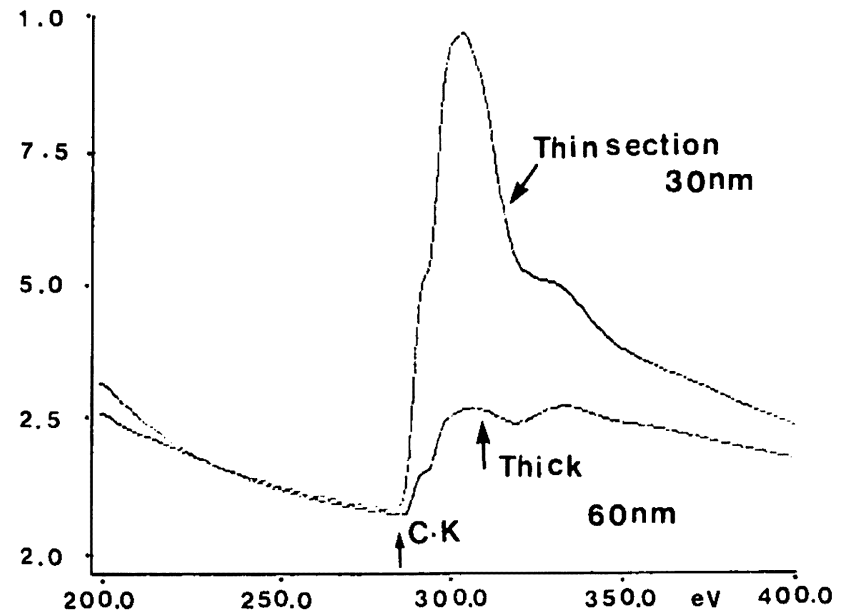

Fig. 2-1. Electron energy-loss spectra shown the correlation between a thick $(60 \mathrm{~nm})$ and thin $(30 \mathrm{~nm})$ pure Epon resin section in the carbon electron energy absorption range at $304 \mathrm{eV}(\mathrm{Max})$ spectra. A $30 \mathrm{~nm}$ section gives a very sharp absorption spectrum with a large amount of electron energy-loss spectroscopic information, but a $60 \mathrm{~nm}$ thicker section does not. Therefore, for ESS analysis, it is necessary to cut ultrathin sections as thin as $30 \mathrm{~nm}$ to get good ESS data. The arrow $C \cdot K$ indicates the absorption edge against carbon (C.K) at $284 \mathrm{eV}$.

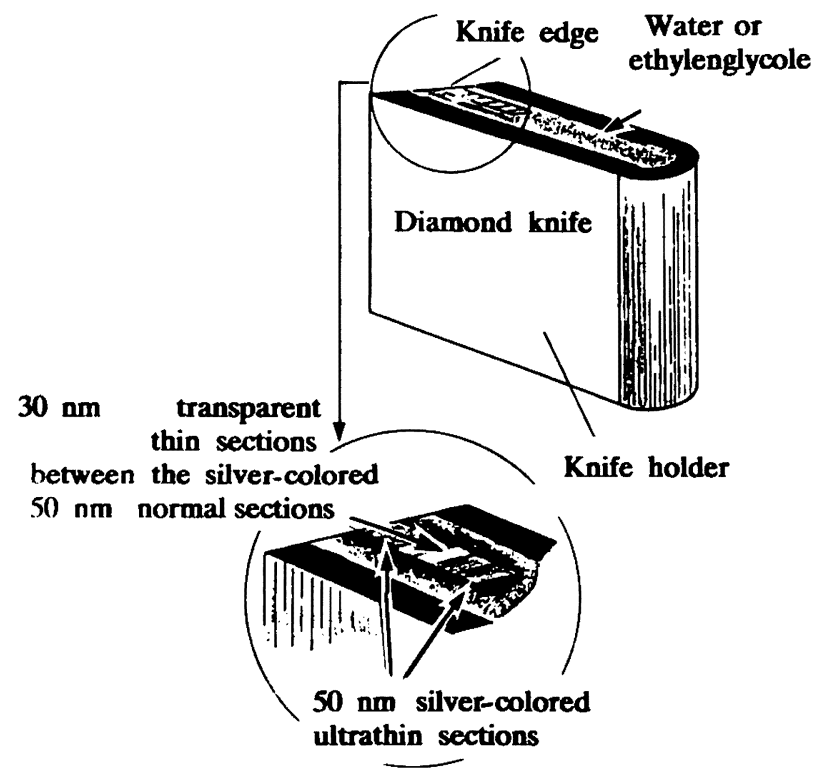

Fig. 2-2. How to cut extremely thin sections for ESS analysis. (1) First, cut two or three silver-colored ultrathin sections at $50 \mathrm{~nm}$ with a diamond knife with a water-bath (for freeze-substitution, use ethylenglycole or glycerine instead of water). (2) Adjust the thickness with the microtome scaler to $30 \mathrm{~nm}$ and, cut the sections carefully. These sections do not show interference color, but are transparent and not visible under a binocular microscope. (3) Next adjust the microtome scaler to $50 \mathrm{~nm}$ and cut some silver-colored sections behind the $30 \mathrm{~nm}$ sections. (4) Thus, $30 \mathrm{~nm}$ sections can be seen as transparent spaces sandwiched between silver-colored sections. (5) Pick up the sections on a 400 or 600 grid mesh with the silver-colored sections. Observe under an ESS electron microscope after drying without electron staining or carbon evaporation.
A
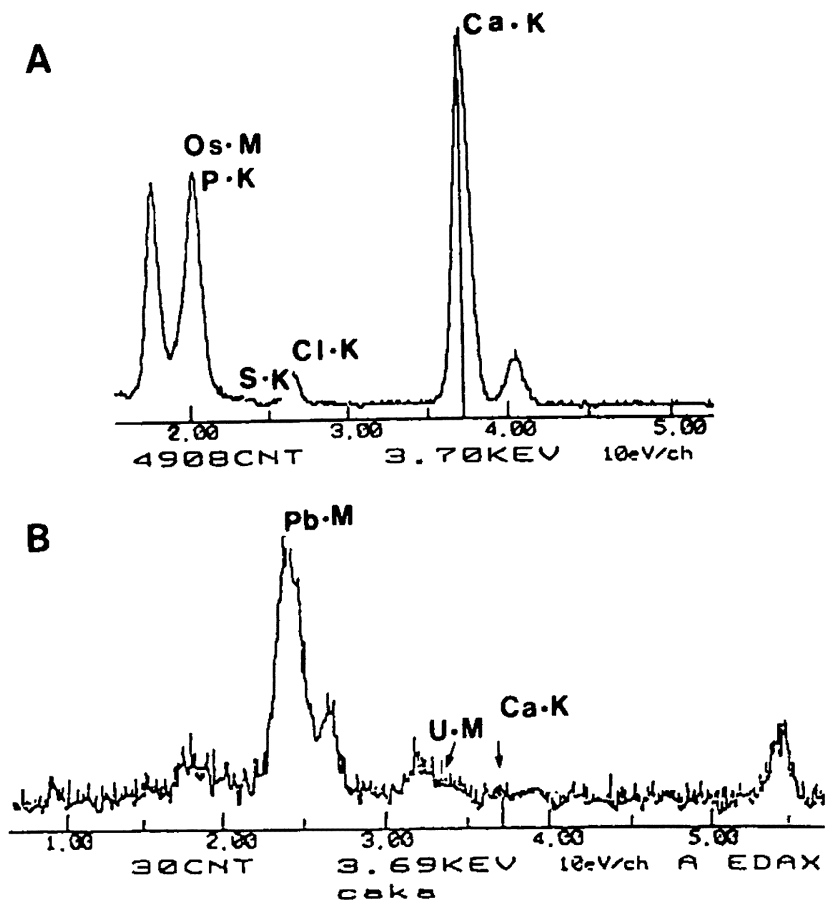

Fig. 3. EDX analyzed spectra obtained from an unstained parietal bone anlage section which was treated with NHA-containing fixative (A), and a double-stained section (B). In A, P.K and Ca.K spectra are very clear, but the $\mathrm{Ca} \cdot \mathrm{K}$ spectrum has disappeared and $\mathrm{Pb} \cdot \mathrm{M}$ and $U \cdot M$ spectra appeared instead after double electron staining with uranyl acetate and lead hydroxide. Calcium was replaced by lead $(\mathrm{Pb})$ or uranium $(\mathrm{U})$ because of ionic balance or ionization tendency. Therefore, even though the electron contrast is very high, there is no calcium left.

section were recorded on a floppy disc (Fig. 9) as digital signals, so as to recognize the distribution of the detected elements (Fig. 1-2 and Fig. 9). Unstained $30 \mathrm{~nm} \mathrm{sec-}$ tions, have no electron contrast, but the electron spectroscopic, dark-field (like) image at about $250 \mathrm{eV}$ helps to show the relations between the cell ultrastructures and elemental distribution in the cell (Fig. 10A, C, E).

Net elemental images were superimposed on the darkfield digital image which was read from the disc. The data stored on a floppy disc were read out on the monitor tube, and analyzed with the Zeiss image analyzer.

Elementally analyzed data were printed out after their background images were subtracted by the image analyzer, and net elemental images were given artificial color with the computer, as follows: net calcium $(\mathrm{Ca} \cdot \mathrm{L})$ image at $355 \mathrm{eV}$ (Max) was expressed with blue color, phosphorus (P.L) at $154 \mathrm{eV}$ with vermillion, oxygen $(\mathrm{O} \cdot \mathrm{K})$ at $544 \mathrm{eV}$ with yellow, sulfur (S.L) at $200 \mathrm{eV}$ with light green, and nitrogen $(\mathrm{N} \cdot \mathrm{K})$ at $410 \mathrm{eV}$ with purple $[10,18,21,23,24]$. Usually, colored net elemental images were superimposed on their dark-field images at $250 \mathrm{eV}$. This is the best way to understand elemental distribution images on the ultrastructures. 


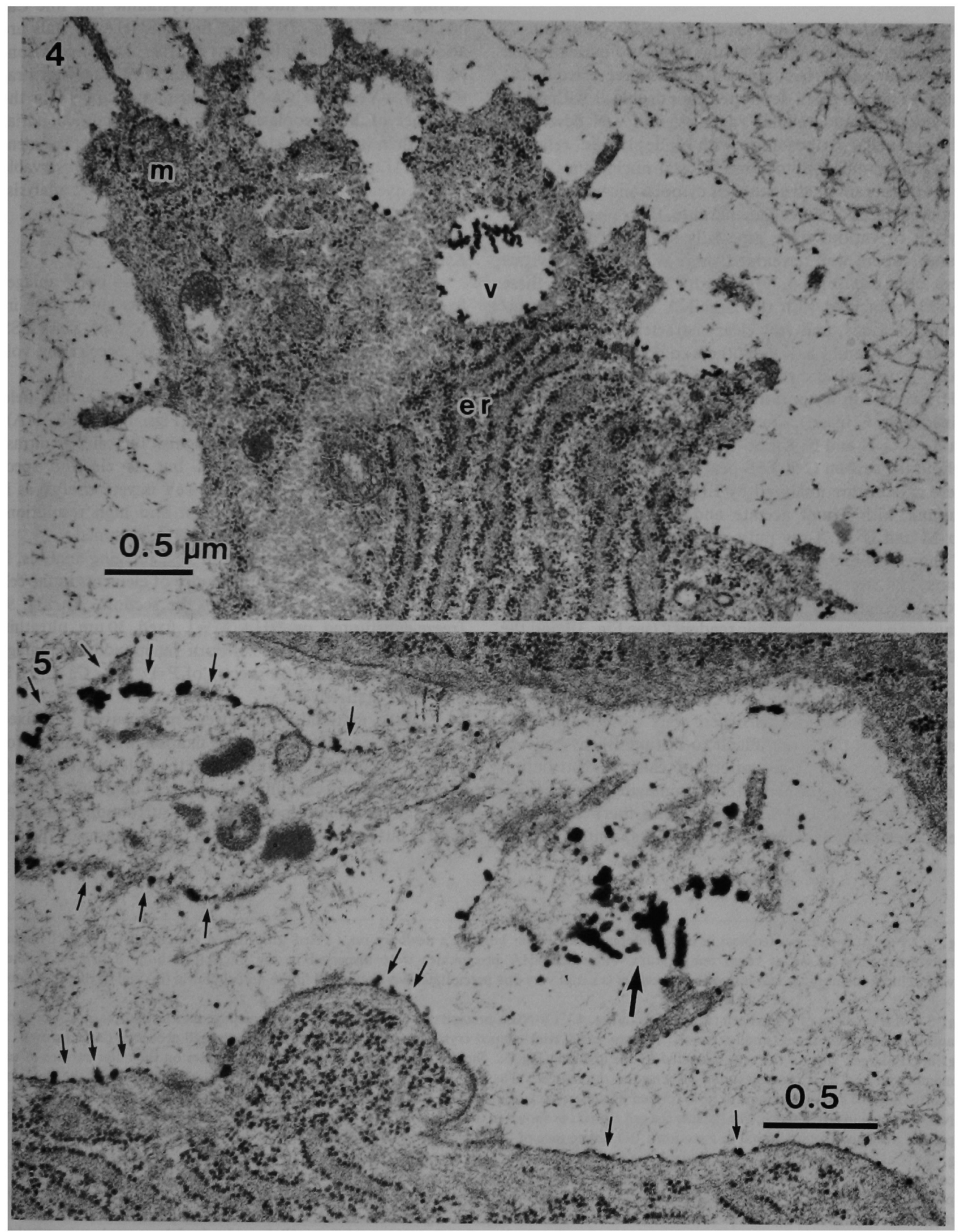

Figs. 4, 5 


\section{Results}

\section{Conventional electron microscopy and EDX analysis}

In the developing mandibular bone and parietal bone anlages, the center is composed of primitive osteoid cartilage. There are well developed chondroblasts with many cell processes and cellular vacuoles, and well developed cell organellae, ribosomes with endoplasmic reticula, glycogen granules, mitochondria, and nuclei (Figs. 4-6). In the membranous structures of cytoplasmic vacuoles and on the surfaces of the cell membranes, there were Ca-NHA crystals of various sizes, especially in the vacuolar membranes, where well developed short stick-shaped crystals were observed (Fig. 4, v). In the EDX analysis, these crystals coincided with the Ca-NHA crystals in the unstained sections, and very clear characteristic X-ray pulse spectra, $\mathrm{P} \cdot \mathrm{K}$ and $\mathrm{Ca} \cdot \mathrm{K}$ and others could be obtained (Fig. 3A). A typical electron diffraction pattern from a $\mathrm{Ca}$ NHA crystal in an unstained section could be easily obtained (Fig. 6, insert). In the ESS-analysis of this crystal, $\mathrm{Ca} \cdot \mathrm{L}_{2,3}, \mathrm{O} \cdot \mathrm{K}$ and $\mathrm{N} \cdot \mathrm{K}$ ESS-spectra were clearly observed behind the carbon C.K ESS-peak (Fig. 1-2). However, if these crystals are analyzed by EDX after double electron staining with uranil acetate and lead hydroxide, clear $\mathbf{P b} \cdot \mathbf{M}$ and $\mathrm{U} \cdot \mathbf{M} \mathbf{X}$-ray pulse spectra are detected instead of the $\mathrm{Ca} \cdot \mathrm{K}$ and $\mathrm{P} \cdot \mathrm{K}$ spectra, even though the electron image contrast was good. During the electron staining procedure, calcium was replaced completely by lead or uranium because of the ionic tendency (see Fig. 3B and the legend of Fig. 6).

Very fine precipitates were scattered in the cytoplasm, ribosomes, mitochondria, and Golgi apparatus, and in the free spaces around the cells there were loose collagenous networks, but they are difficult to distinguish by conventional observations (the pattern of calcium distribution can only be distinguished after ESS-imaging; see Figs. 10, 11). In the developing mandibular bone, there can be seen well developed fine collagenous networks, matrix vesicles close to the osteoblasts, and the beginning of many calcifying centers with fine apatite crystalline and fine $\mathrm{Ca}$ NHA crystals (Fig. 7). Very fine Ca-NHA crystals are also scattered in the collagenous matrix. For comparison, we show a section from cardiac muscle of a newborn rat (Fig. 8). Very fine Ca-NHA precipitates appear in the endosack of the sarcoplasmic reticulum and mitochondria, better than in the two-step oxalate-antimony replacement method [12, 16, 19, 20, 23]. This is not easily observable with conventional electron microscopy or EDX analysis, but it is possible with ESS-imaging.

\section{ESS-imaging analysis}

Ultrathin sections from newborn rat bone anlages fixed with NHA-containing fixative under microwave irradiation (MWI) were observed with a Carl Zeiss ESS electron microscope LEO-912 at $100 \mathrm{kV}$ accelrating voltage (Okayama University).

The 0-loss image or electron spectroscopic, dark-field image at $250 \mathrm{eV}$ was observed first (Figs. 10A, C, E, 11A). The 0-loss image could not distinguish the cellular ultrastructures in biological sections, but an electron spectroscopic, dark-field image at $250 \mathrm{eV}$ is very useful, as it shows very high electron contrast and high resolution, even when the section is very thin and unstained.

Prior to the development of ESS-imaging analysis, it was neccesary to detect the existance of elements in the cell by taking the ESS-spectra from the section. In Fig. 9, freeze-substituted or NHA/MWI fixed $30 \mathrm{~nm}$ ultrathin section obtained from a newborn rat mandibular anlage show very excellent ESS-spectra of $P \cdot L, S \cdot L, C a \cdot L, N \cdot K$ and $\mathrm{O} \cdot \mathrm{K}$, respectively.

In the materials which were microwaved and fixed with NHA-containing fixative, fixation was excellent for viewing calcium ion distribution in the cells and tissues. A large amount of calcium was diffusely distributed in the active chondroblast endoplasm and surrounding cartilage matrix but could not be found in the exoplasm of the cell

Fig. 4. Part of a young osteoblast in the developing mandibular bone of a newborn rat. Fixed with fixative containing $0.1 \mathrm{mM}$ NHA and microwaved for $35 \mathrm{sec}$ at room temperature. Fine Ca-NHA precipitates were dispersed in the uncalcified bone matrix, associated with cellular membranes and intracellular vesicles $(v)$, and very fine precipitates were observed in the mitochondria (m) and in the ribosomes. $\times 30,000$.

Fig. 5. Rib cartilage of a newborn rat, fixed as in Fig. 4. Ca-NHA precipitates were dispersed in the cartilage matrix, cytoplasmic processes, and their cell membranes (small arrows). In some cases, stick-shaped crystals formed inassociation with the cell processes (large arrow).

Fig. 6. Enlarged view of the area indicated by the arrow in Fig. 5. Small arrows indicate Ca-NHA precipitates on the outer surface of cell membranes and their processes, and dispersed in the matrix. The growth of Ca-NHA crystals is related with the density of the Ca ${ }^{2+}$ ion distribution. The crystal seems to have formed in a short stick shape, which indicates the C-axis of the crystal, if both ionic elements, Ca and NHA, existed in a free space, like outside the cell matrix, as shown in the electron diffraction pattern (the inserted shows an electron diffraction pattern obtained from a crystal in a section. 011 lattice face of the reciprocal lattice is shown).

Fig. 7. Calcifying newborn rat mandibular bone matrix. In the upper right corner, elongated cell processes and matrix vesicles of an osteoblast can be seen, and on the left side, the beginning of the nuclei of calcium deposition on the fine collagenous networks. Very fine Ca-NHA precipitates dispersed in the bone matrix. Those calcifying nuclei were different from matrix vesicles, as they did not surrounded by the membranous structures.

Fig. 8. Newborn rat cardiac muscle, fixed as described in Fig. 4. Very fine Ca-NHA precipitates fill the endsack of the sarcoplasmic reticulum (arrow). t, T-tubule; z, Z-band; gly, glycogen granules; $m$, mitochondria. Ca-NHA precipitates have little electron opacity, so that they are not easily differentiated in normal electron microscopy. 


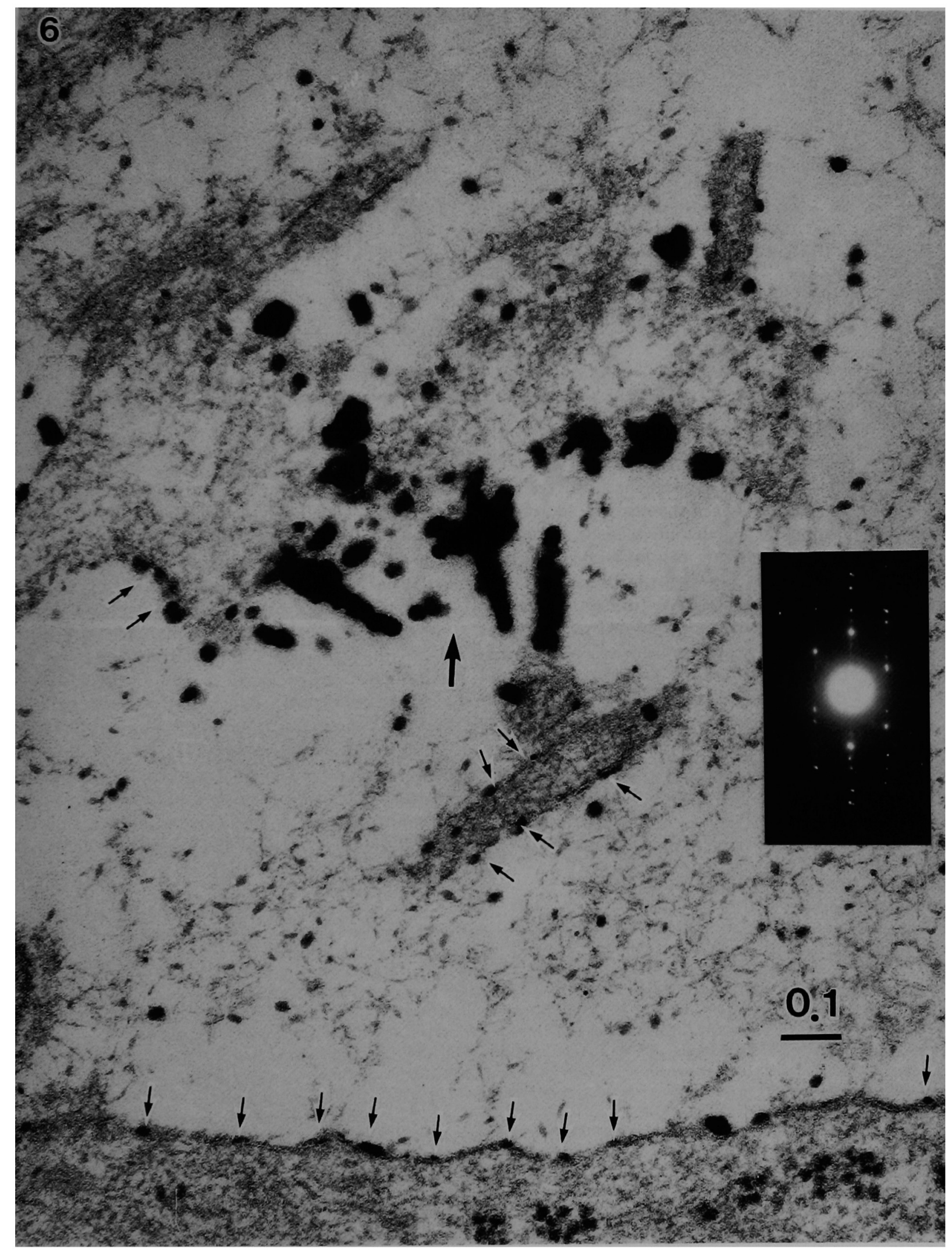

Fig. 6 


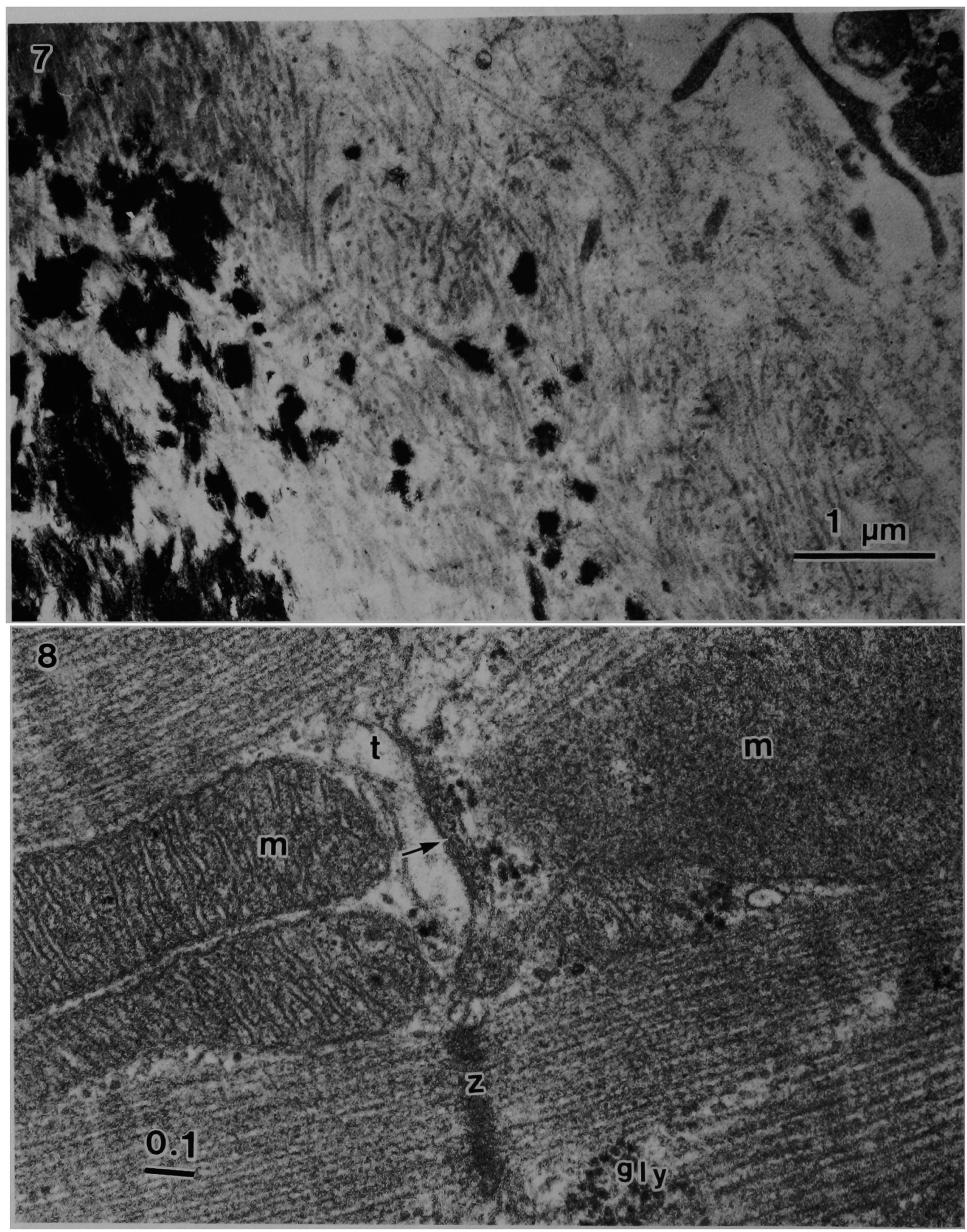

Figs. 7, 8 


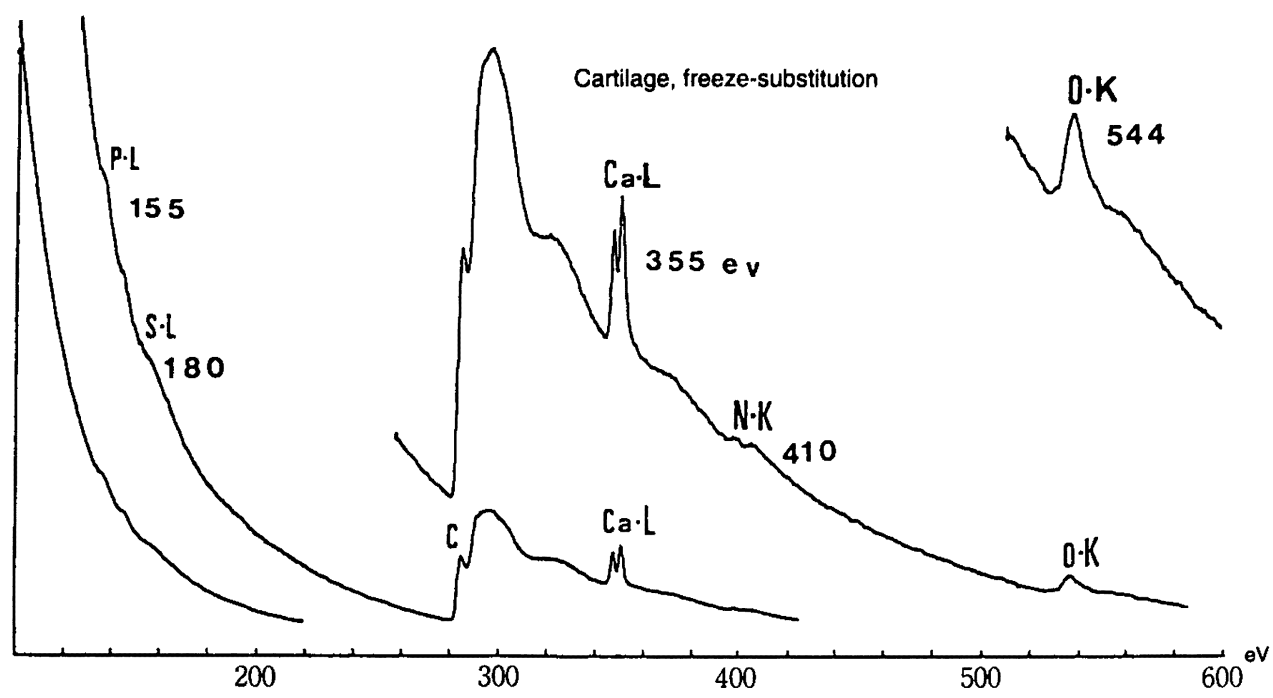

Fig. 9. ESS spectra obtained from newborn rat mandibular bone anlage (cartilage ossification) in a freeze-substituted $30 \mathrm{~nm}$ section. $P \cdot \mathbf{L}$, S.L, Ca.L, N.K and O.K energy absorption spectra are very clear [10]. Taken with a Zeiss EM-902A (University of Niigata, Dental School).

at lower magnification (Fig. 10B). In the partially enlarged view of the cell endoplasm in Fig. 10B, a large amount of calcium was observed in the cellular ultrastructures, endoplasmic reticula with ribosomes, mitochondria, Golgi membrane, and nuclear chromatins, but no calcium was observed in the exoplasm (expl). Such results have never been observed by the oxalate/antimony method. The results were similer to those obtained with frozen sections or the freeze-substitution method.

We also analyzed some mesenchymal and endothelial cells far from the calcifying region. Only a little calcium was found in the endothelial cells of capillaries, and very little was found in the mesenchymal cells (Fig. 10E, F).

In part of a young osteoblast in a newborn rat mandibular anlage, there are well developed cell organellae: endoplasmic reticula with ribosomes, Golgi apparatus, mitochondria, small vesicles, and nuclear chromatins (Fig.
11A). A large amount of calcium is distributed throughout the cell endoplasm, on the endoplasmic reticula, ribosomes, mitochondria and nuclear chromatins (Fig. 11B), but there is no calcium in the exoplasm. Other cell elements were also analyzed, and the data showed good images of nitrogen, $\mathrm{N} \cdot \mathrm{K}$ (Fig. 11C), oxygen, $\mathrm{O} \cdot \mathrm{K}$ (Fig. 11D), sulfur, S.L (Fig. 11E), and phosphor, P.L (Fig. 11F). The location of these elements was in good correspondence with the cellular ultrastructures except for sulfur in ribosomes. Ribosomes show negative images along the endoplasmic reticulum (Fig. 11E). For example, the cell membranes, ribozomes, endoplasmic reticula, mitochondrial membranes, Golgi membranes, and nuclear chromatins are shown. The existance of the elements $\mathrm{N}$, $\mathrm{O}, \mathrm{S}, \mathrm{P}$ and $\mathrm{Ca}$ is reasonable because the cell ultrastructures are composed of proteins.

Fig. 10. Newborn rat mandibular bone anlage, fixed with MWI for $35 \mathrm{sec}$ at room temperature with aldehyde fixative containing $0.1 \mathrm{mM}$ NHA. Observed under a Zeiss LEO-912 microscope (Okayama University). A: Part of a chondroblast in a lacuna (lac). A large amount of the exoplasm (expl) and cellular ultrastructures are condensed in the endoplasm (endpl) surrounded by an external cell bank. $250 \mathrm{eV}$ dark-field image. B: Net Ca.L ESS-image at $355 \mathrm{eV}$ after the background image was subtracted by image analyzer. Calcium (blue dots) is distributed in the endoplasm, outside cell bank, lacuna and matrix, at low magnification. C: Enlarged view of part of the endoplasm of Fig. 10A. Well developed mitochondria (m), endoplasmic reticula with well developed ribosomes, and a nucleus (n) can be seen. D: Calcium (Ca.L) net image distribution in the endoplasm of $\mathbf{C}$, at $355 \mathrm{eV}$ (blue dots). A large amount of calcium is distributed in the endoplasm, mitochondria, ribosomes, endoplasmic reticula, and nucleus, but not in the exoplasm. E: Part of a capillary endothelial cell (arrow) and associated mesenchymal cell far from the calcifying region, $250 \mathrm{eV}$ image. F: A small amount of calcium was observed in the endothelial cell, but very little was observed in the mesenchymal cell.

Fig. 11. Part of an active chondroblast in a newborn rat mandibular anlage, observed under a Zeiss LEO-912 ESS-electron microscope (Okayama University). A: Part of the endoplasm with well developed Golgi apparatus (g), endoplasmic reticula with well developed ribosomes, mitochondria (m), and nucleus (n), at $250 \mathrm{eV}$ in a dark-field image. B: A calcium net image (Ca. $\mathrm{L}$, blue dots) was superimposed on the original $250 \mathrm{eV}$ image. A large amount of calcium was distributed in the Golgi, ribosomes, endoplasmic reticula, mitochondria, and nucleus. C: A net nitrogen (N.K, purple dots) image at $410 \mathrm{eV}$ was superimposed on the $250 \mathrm{eV}$ background image. D: A net oxygen (O.K, yellow dots) at $544 \mathrm{eV}$ image was superimposed on the $250 \mathrm{eV}$ image. E: A net sulfur (S. L, light green dots) ESS-image at $200 \mathrm{eV}$ was superimposed on the $250 \mathrm{eV}$ image. Ribosomes show negative images (white dotts) in regard to sulfur. F: Distribution of net phosphorus (P.L, vermilion dots) at $154 \mathrm{eV}$, not superimposed on the $250 \mathrm{eV}$ dark-field background image. The elemental images corresponded to the cellular ultrastructures and the natures of their proteins. 

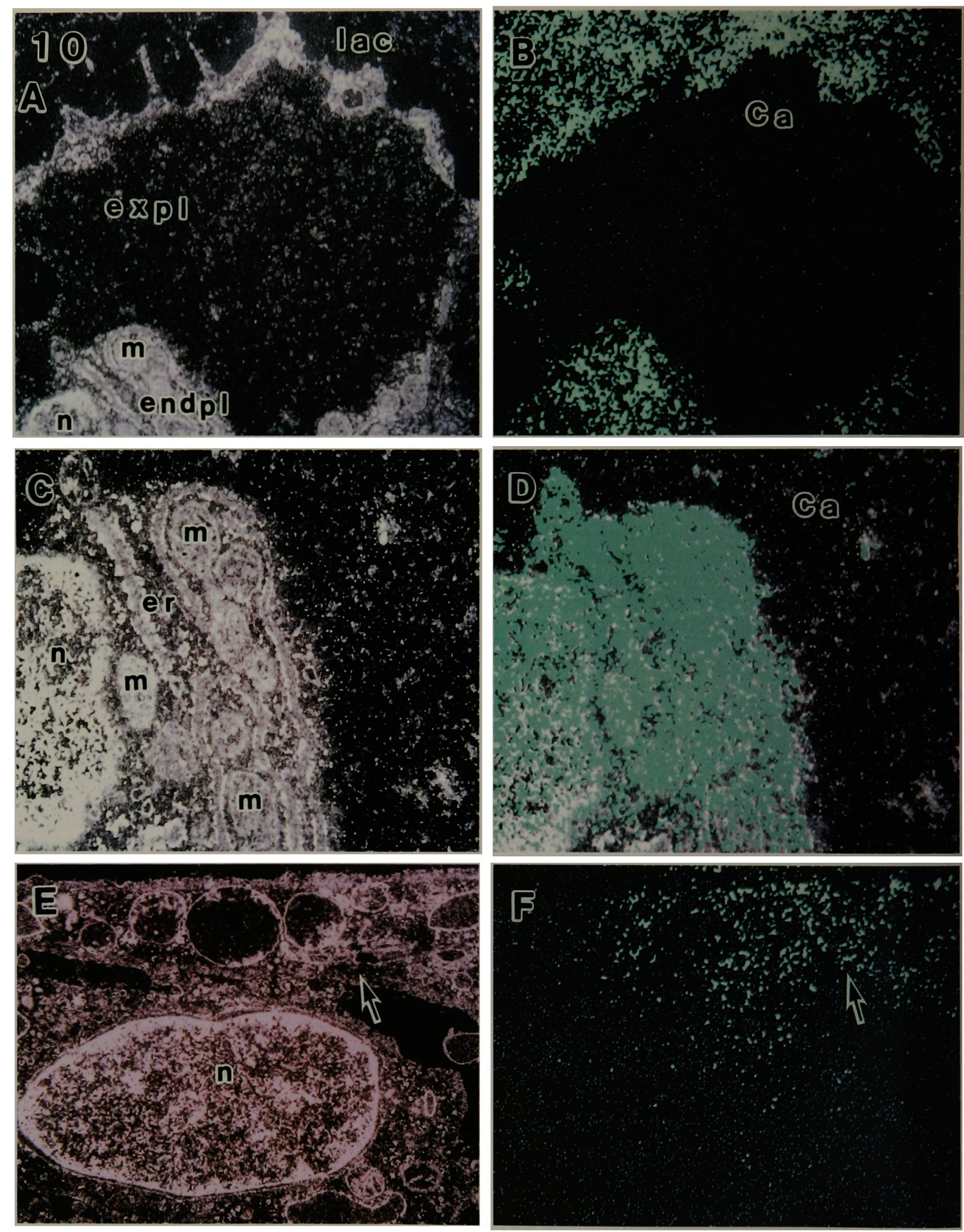

Fig. 10 

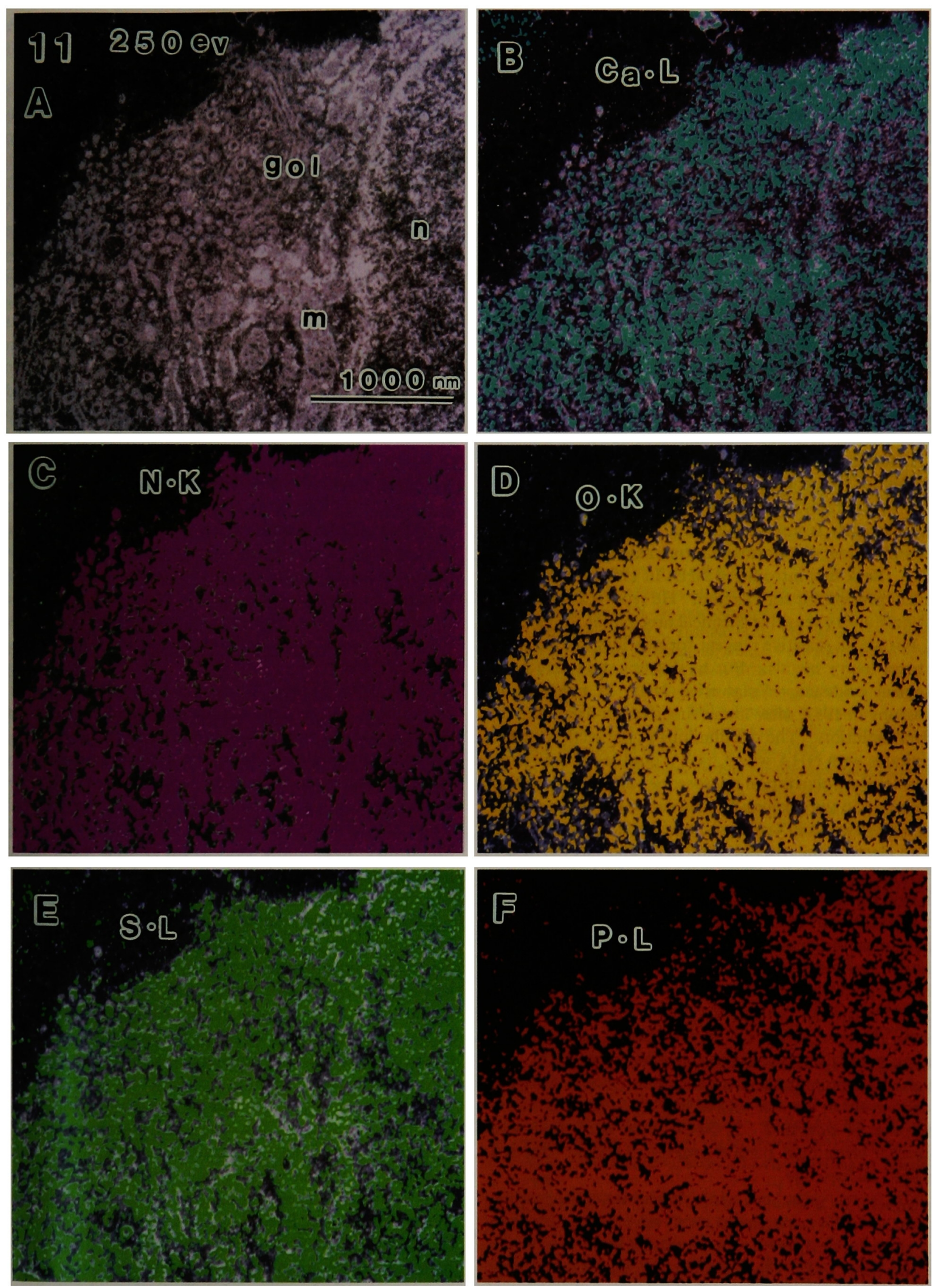

Fig. 11 


\section{Discussion}

In our previous reports concerning the detection of $\mathrm{Ca}^{2+}$ ions in the cell $[14-16,18,20,21]$, we recommended a two-step replacement method with oxalate/antimony inorganic precipitation with microwave fixation, as the best method after frozen dry or freeze-substitution methods [ 9 , $10,12,16,18,21,23]$. This time, however, we discovered that a small amount of NHA in the fixative is a very sensitive and reliable precipitant for $\mathrm{Ca}^{2+}$ ions with microwave fixation and analysis with ESS-imaging, so as to be equal to the frozen dried or freeze-substitution method [24].

The discussion can be divided into several points: (1) problems of the chemical sensitivity to $\mathrm{Ca}^{2+}$ ions and solubility in the solvents, (2) electron opacity of the CaNHA precipitates, (3) fixation methods, especially the efficiency of combined MWI fixation, (4) and the analysing methods, conventional electron microscopy, computerized EDX analyzing, and ESS-imaging.

(1) Regarding the chemical sensitivity of NHA against $\mathrm{Ca}^{2+}$ ions, $0.1 \mu \mathrm{g} / \mathrm{ml}[1]$, produces very fine and very insoluble precipitates in water or organic solvents. (2) The electron opacity is not so high, but the precipitates were still visible under the normal electron microscope [12, 19, 21, 24]. Beck first introduced NHA in 1951 as a excellent chemical precipitant for calcium. He reported that NHA is not only a very sensitive chemical precipitant for calcium ions, but also a sensitive reagent for peptides and proteins and some heavy metals. But he did not show any morphological evidence. Voigt tried NHA in polarization microscopy in 1957, after immersing alcohol-fixed slices in an NHA solution. The calcium ions in the sections made precipitates with NHA which have a double-refractive nature under the polarization microscope [40]. Przelęcka, et $a l$. reported on the calcium distribution in the insect oocyte using a SEM and EDX analysis, and Zechmeister in 1979, used an electron microscope with EDX analysis [34, 42].

Unfortunately, their sampling methods, especially their fixation methods with NHA were unsuitable. They used conventional fixation with aldehyde first, and these tissue blocks were immersed in the NHA solution. In this method, most soluble calcium ions would be leached out into the fixative before the NHA treatment, although some remained in the cell, where they were bound with proteins or some glycoproteins. The idea proposed by Voigt [40] of using polarization microscopy seems to be good, even though his preparation methods were poor. If fresh material could be treated with MWI/NHA fixation, better data could be obtained. If NHA could be bound with fluorescent chemicals, further progress could be expected with light microscopy.

Almost ten years ago, while developing our new fixation method MWI, we found that NHA was better than other chemical precipitants for demonstrating $\mathrm{Ca}^{2+}$ ions in cells and tissues $[12,19]$. In the past, we observed very fine precipitates associated with the cell membranes, intracellular vacuoles, and developing young bone or car- tilage matrix. Fine crystals were grown to their $\mathbf{C}$-axis in the form of a stick, which coincided with its electron diffraction pattern (Fig. 6, insert).

However, at that time, we did not have enough data on the fixation and distribution of calcium in a cell, and using EDX analysis [12,16,19, 21], we concluded that oxalic acid/antimony replacement was the best method for demonstrating the localization of calcium in a cell, after the freezing methods $[12,14-16,18-21,23]$. Recently, we had the chance to analyze the same sections which were fixed with NHA-containing fixative and MWI, observed with a Carl Zeiss LEO-912 ESS electron microscope. The cell ultrastructures were fixed well, and a large amount of calcium remained in the cell, even though the precipitates could not be easily recognized under normal electron microscopy. In a highly magnified ESS-image, the net elemental image correctly coincided with the cellular ultrastructures, Golgi, mitochondrial membranes, ribosomes, and nuclear membranes or chromatins, in $N \cdot K$, $\mathrm{O} \cdot \mathrm{K}, \mathrm{P} \cdot \mathrm{L}, \mathrm{S} \cdot \mathrm{L}$, and $\mathrm{Ca} \cdot \mathrm{L}$ distribution respectively. In this experiment, the distribution of calcium is the most important problem: large amounts exist in cell endoplasm including cellular ultrastructures, and in the surrounding bone matrix, except in the cell exoplasm where there is a large deposit of glycogen, in the newborn rat calcifying tissues. In the cell which are not concerned with the ossificating functions, free cells in the mesenchymal tissue did not show calcium accumulation in the cell cytoplasm (Fig. 10E, F).

The NHA method for calcium ion detection seems to be the best way to demonstrate calcium distribution for electron microscopy, when the ESS-imaging method is used. For fixing $\mathrm{Ca}^{2+}$ ions, the combination of microwave fixation with the use of tannic acid (TA). NHA seems to produce good results with TA, because NHA also reacts with cellular proteins to produce good fixation [1]. Microwave irradiation causes rapid and homogenous infiltration of chemicals including fixative $[12,14-16,18-$ 23].

ESS analysis for biological specimens was introduced by Ottensmeyer and his coworkers including the theoretical background for the applications [28-32]. Probst [33] and Rahmann and his coworkers $[5,6,35]$ reported detection of calcium or calcium related enzymes with the ESSimaging method, but they did not touch on fundamental problems, such as how to detect $\mathrm{Ca}^{2+}$ ions or sensitivity for the detection of calcium ions.

The most important and characteristic merit of the ESS imaging analysis for biological use seems to be the demonstration of the elemental distribution as a continuous high resolution image in the cellular ultrastructures, with a very high quality, dark-field electron spectroscopic image. Of course, ESS analysis has other good potentials, such as the possibility of a 0 -loss image, and this gives very high quality electron diffraction patterns and very important chemical structural information about organic chemicals, but it is not useful for very thin $(30 \mathrm{~nm})$ bio- 
logical unstained sections, as mentioned above. For ultrathin biological sections, electron spectroscopic images at about $250 \mathrm{eV}$ are more useful than the 0-loss images. Of course, the ESS method is also very useful, to obtain very sharp, high resolution and good contrast images for thicker sections of $0.5-1 \mu \mathrm{m}$ or more. But this is characteristic not only of the ESS electron microscopy but also of high tension electron microscopy.

Thus, this NHA/MWI/ESS combination method is the best way to demonstrate the distribution of elements such as calcium ions in cells and tissues, and we hope to use for other important tissues, such as muscle and nervous tissues.

We tried to demonstrate the distribution of analyzed elements with artificially colored digital images superimposed on the original monochronal dark-field (at $250 \mathrm{eV}$, electron spectroscopic image) image. Originally, it was difficult to distinguish the distribution sites in the cell organellae in the monochromal display. However, when the elemental image was colored by the computer of the image analyzer, and superimposed on the original dark-field image, it became very easy to understand the correlation between the sites of elemental distribution and the cellular ultrastructures $[10,18,23]$ (Figs. 10A/B, C/D, E/D, $11 \mathrm{~A} / \mathrm{B}, \mathrm{C}, \mathrm{D}, \mathrm{E}, \mathrm{F})$. In particular, when imaging sulfur, we found that ribosomes appeared as negative images in the cell cytoplasm associated with the endoplasmic reticulum, because ribonucleic acid does not contain sulfur (Fig. 11E).

Carl Zeiss ESS electron microscopes have two electron spectroscopic systems, having a prism/mirror/prism type electron spectrometer, EM-902A, and a further-developed Omega-type spectrometer, and an image analysing system, CEM, or an IBAS system. The Omega type, which we call the LEO-912 electron microscope, is easy to operate and can produce very high quality ESS-images. We obtained very good results with the EM-902A, but this time we tried the LEO-912 for newborn rat calcifying tissues, and obtained excellent results.

The next important problem will be the ESS analysis of elements in quantitative calculations, localization, and the movement of elements in and out of the cell. There is also the possibility of application to studies of genetics or its transformations using labelled rare elements in biological tissues, such as selenium (Se), scandium (Sc), cesium (Cs), or bromine (Br), for the ESS analysis, as tried under EDX before [9]. It is possible to calculate the volume of existing-elements quantitatively, in sections of constant thickness, after the thickness is correctly determined. The analytical resolution can be expected to be in the range of a few atoms per ultrathin section.

\section{Acknowledgments}

We thank Miss E. Takagawa, Carl Zeiss Vision Co., Ltd., Tokyo, for her effective technical assistance for ESS-imaging by the image-analyzer operations. We also thank Dr. R. Goris for proofreading the manuscript.

\section{References}

1. Beck, G.: Oxydimetrische titration in alkalischen lösungen mit kaliumcuri-3-perjodat (Pereupsimtrie). Mikrochemie. 36/37; 245-250, 1951.

2. Bogers, M., Thoné, F, and Van Nueten, J. M.: The subcellular distribution of calcium and the effects of calcium-antagonists as evaluated with a combined oxalate-pyroantimonate technique. Acta Histochem. (Suppl.) 24; 327-332, 1981.

3. Bogers, M., Thoné, F., Verheyen, A. and Keurs, H. E. D. J.: Localization of calcium in skeletal and cardiac muscle. Histochem. J. 16; 295-309, 1984.

4. Komnick, H.: Elektronmikroskopische localization von $\mathrm{Na}$ und $\mathrm{Cl}$ in zellen and geweben. Protoplasma 55; 414-422, 1962.

5. Körtje, K. H., Freihöfer, D. and Rahmann, H.: Cytochemical localization of calcium in the central nervous system of vertebrates. Ultramicrosc. 32; 12-17, 1990.

6. Körtje, K. H., Freihöfer, D. and Rahmann, H.: Cytochemical localization of high affinity Ca-ATPase activity in synaptic terminals. J. Histochem. 38; 895-900, 1990.

7. Legato, M. J. and Langer, G. A.: The subcellular localization of calcium ions in mammalian myocardium. J. Cell Biol. 41; $401,1969$.

8. Mata, M., Steple, J. and Fink, D. J.: Ultrastructural distribution of $\mathrm{Ca}$ within neurons. An oxalate pyroantimonate study. Histochemistry 87; 339-349, 1987.

9. Mizuhira, V.: Elemental analysis of biological specimens by electron probe X-ray microanalysis. In "Pure and Applied Chemistry", ed. by T. Tsuruta, et al., Vol. 50: A1-19, Chemistry for the Welfare of Mankind, Congr. Pure and Applied Chemistry, Pergamont Press, Tokyo, 1978.

10. Mizuhira, V.: Recent advances in biomedical microbeam analysis: -From EDX to EELS-Imaging analysis of biomedical specimens, especially with respect to calcifying tissue. J. Electron Microsc. 38 (Suppl); S142-S146, 1989.

11. Mizuhira, V.: Fixation method for proteins using tannic acid, Current Content. LS.V.35,\#24, June 15, 1992, p. 9.

12. Mizuhira, V.: New Tissue Fixation and Staining Methods by the Aid of Microwave Irradiation. Gakusai-Kikaku Press, Tokyo 1993. (in Japanese)

13. Mizuhira, V.: Autoradiography for Biomedical Research, -From Macroscopic Autoradiography to Electron Microscopic Autoradiography-, Gakusai Kikaku Press, Tokyo, 1993. (in Japanese)

14. Mizuhira, V.: Microwave fixation method for cytochemistry for conventional electron microscopy, enzymo- immunocytochemistry, autoradiography and elemental distribution studies by means of EDX and EELS-Imaging analysis-. 10th Internat. Congr. Histochem. Cytochem., Proc., Kyoto, 1996, pp. 315-316.

15. Mizuhira, V.: Microwave fixation method for cytochemistry for conventional electron microscopy, enzymo- immunocytochemistry, autoradiography, elemental distribution studies and staining method using microwave irradiation-. XXIth Internat. Congr. \& Internat. Acad. Pathol. \& 12th World Congr. Acad. Environment. Pathol., Budapest, Hungery, Handout, 1996.

16. Mizuhira, V.: Microwave fixation method for cytochemistry. -For conventional electron microscopy, enzymo- immunocytochemistry, autoradiography, elemental distribution studies and staining methods. Europ. J. Morphol. 34; 385-391, 1996.

17. Mizuhira, V. and Ueno, M.: Calcium transport mechanism in molting cryfish revealed by microanalysis. J. Histochem. Cytochem. 31; 214-218, 1983. 
18. Mizuhira, V. and Hasegawa, H.: Microwave fixation and localization of calcium in synaptic terminals by means of the $\mathrm{X}$ ray microanalysis (EDX) and electron energy loss spectroscopy (EELS) imaging methods. Brain Res. Bull. 43; 53-58, 1997.

19. Mizuhira, V., Notoya, M. and Hasegawa, H.: New tissue fixation method for cytochemistry using microwave irradiation. I. General remarks. Acta Histochem. Cytochem. 23; 501-523, 1990.

20. Mizuhira, V., Hasegawa, H. and Notoya, M.: Microwave fixation for histo- and cytochemistry. In "Electron Microscopic Cytochemistry and Immunocytochemistry in Biomedicine", ed. by K. Ogawa and T. Barka, CRC Press, N.W.Fl., USA, 1993, pp. 17-27.

21. Mizuhira, V., Hasegawa, H. and Notoya, M.: Localization of calcium in synaptic vesicles. $J$. Neurosci. Methods 55; $125-$ 136, 1994.

22. Mizuhira, V., Notoya, M. and Hasegawa, H.: Demonstration of steroid and peptide hormone receptors by electron microscope autoradiography, In "Autoradiography and Correlative Imaging", ed. by W. E. Stumpf and H. F. Solomon, Academic Press, New York, 1995, pp. 497-513.

23. Mizuhira, V., Hasegawa, H. and Notoya, M.: Microwave fixation and localization of calcium in synaptic terminals and muscular cells by electron probe X-ray microanalysis and electron energy-loss spectroscopy imaging. Acta Histochem. Cytochem. 30; 277-301, 1997.

24. Mizuhira, V., Hasegawa, H., Akagi, T. and Nagai, N.: Ca ion distribution in calcifying tissue analyzed with Zeiss LEO-912 electron spectroscopic (ESS) imaging under microwaved fixation in a NHA containing fixative. 5th US-Japan Joint Congr. of Histochem. Cytochem. Soc., Univ. of California, San Diego, 1998.

25. Notoya, M., Hasegawa, H. and Mizuhira, V.: New tissue fixation method for cytochemistry using microwave irradiation. II. Details. Acta Histochem. Cytochem. 23; 525-536, 1990.

26. Ogawa, S., Kashiwagi, A., Kikkawa, R. and Shigeta, Y.: Increase of Voltage-sebsitive calcium channels and calcium accumulation in skeletal muscles of Streptozocin-induced diabetic rats. Metabolism 44; 1455-1461, 1995.

27. Ohara, P. T., Wade, C. R. and Lieberman, A. R.: Calcium storage sites in axon terminals and other components of intact CNS tissue: studies with a modified pyroantimonate technique. J. Anat. 129; 869-870, 1979.

28. Ottensmeyer, F. P.: Electron microscopic imaging: Parallel energy filtering and microanalysis in the fixed beam electron microscope. J. Ultrastruct. Res. 88; 121-134, 1984.
29. Ottensmeyer, F. P.: Elemental mapping by energy filtration: Advantages, limitations and compromises. Ann. NY. Acad. Sci. 483; 339-350, 1986.

30. Ottensmeyer, F. P. and Andrew, J. W.: High-resolution microanalysis of biological specimens by electron energy loss spectroscopy and by electron spectroscopic imaging. J. Ultrastruct. Res. 72; 336-348, 1980.

31. Ottensmeyer, F. P., Whiting, F. R., Schmidt, R. S. and Clemens, R. S.: Electron microscopy of proteins. A close look at the ashes of myokinase and protamine. J. Ultrastruct. Res. 52; 193-201, 1978.

32. Ottensmeyer, F. P., Bazett-Jones, D. P., Henkelman, R. M., Korn, A. P. R. and Whiting, R. F.: The imaging of atoms: Its application to the structure determination of biological macromolecules. Chemica Scripta. 14; 257-262, 1978.

33. Probst, W.: Ultrastructural localization of calcium in the CNS of vertebrates. Histochemistry 85; 231-239, 1986.

34. Przelęcka, A., Allakhverdov, B. L., Growaka, S. K. and Pogorelov, A. G.: Ultracytochemical localization and insect oocyte. Histochemistry 85; 163-168, 1986.

35. Rahmann, H.: Brain gangliosides and memory formation. Behav. Brain Res. 66; 105-116, 1995.

36. Simson, J. A. V. and Spicer, S. S.: Selective subcellular localization of calcium with variations of the potassium pyroantimonate technique. J. Histochem. Cytochem. 23; 575-598, 1975.

37. Tachibana, T., Nawa, T., Mizuhira, V. and Yoshida, Y.: Ultrastructural localization of calcium in mechanoreceptors of the oral mucosa. J. Neurocytol. 21; 745-753, 1992.

38. Takaichi, S., Mizuhira, V., Hasegawa, H., Ozawa, H., Ejiri, S. and Notoya, M.: Ultrastructure and calcification of embryonal snail shell. Acta Histochem. Cytochem. (submitted)

39. VanReempts, J., Borgers, M. and Offner, F.: Ultrastructural localization of calcium in the rat retina with a combined oxalate-pyroantimonate technique. Histochem. J. 16; 295-309, 1984.

40. Voigt, G. T.: Ein neuer histotopochemischer Nachweis des Calcium (mit Naphthalylhydroxamsäure), Acta Histochem. (Jena) 4; 122-131, 1957.

41. Wicks, S. M. and Helper, P. K.: Selective localization Ca with potassium antimonate. J. Histochem. Cytochem. 30; 11901204, 1982.

42. Zechmeister, A.: A new selective ultrahistochemical method for the demonstration of calcium using $n, n-n a p h t h a l o y l h y d r o x-$ ylamine. Histochemistry 62; 223-232, 1979. 